\title{
Quasi-static and Dynamic Compression Behaviors of an in-situ Ti-based Metallic Glass Matrix Composite
}

\author{
M.Y. Chu' ${ }^{1}$, Z.M. Jiao' ${ }^{2}$ Z.H. Zhang ${ }^{3}$, Z.H. Wang ${ }^{2}$, H.J. Yang ${ }^{1,4}$ and J.W. Qiao ${ }^{1, *}$ \\ ${ }^{1}$ Laboratory of Applied Physics and Mechanics of Advanced Materials, College of Materials Science and Engineering, Taiyuan University of \\ Technology, Taiyuan 030024, China \\ ${ }^{2}$ Institute of Applied Mechanics and Biomedical Engineering, Taiyuan University of Technology, Taiyuan 030024, China \\ ${ }^{3}$ College of Electrical and Power Engineering, Taiyuan University of Technology, Taiyuan 030024, China
}

${ }^{4}$ Research Institute of Surface Engineering, Taiyuan University of Technology, Taiyuan 030024, China

\begin{abstract}
A kind of in-situ Ti-based metallic glass matrix composite containing ductile dendrites was fabricated. Quasi-static and dynamic compression behaviors of the composite were investigated. Upon quasi-static loading, the composite exhibits ultrahigh strength, accompanied by macroscopic plasticity. In micro, multiple shear bands on the lateral surface and abundant droplets on the fracture surface characterize the plasticity of the composite. However, all the samples fracture with brittleness due to the insufficient time to generate multiple shear bands. The different deformation and fracture mechanism of the composite upon quasi-static and dynamic loading are interpreted.
\end{abstract}

\section{Introduction}

Bulk metallic glasses (BMGs) exhibit a series of excellent mechanical properties at ambient temperature, such as high strength, large elastic limit, and superior corrosion and wear resistance [1], which render them the potential as structural materials. However, almost all BMGs suffer from a fatal problem that they exhibit catastrophic failure without obvious macroscopic plasticity upon loading at room temperature, due to the prompt propagation of highly localized shear bands [2]. In order to dispose of the problem, a variety of methods have been applied recently. One effective method is to introduce a second phase with ductility into the glass matrix to fabricate metallic glass matrix composites (MGMCs) [3-8]. The second phase works as an obstacle to prevent the prompt propagation of shear bands and facilitate their multiplication, avoiding an early failure of the composites upon loading. Consequently, improved plasticity is available for MGMCs. Up to now, there are mainly three ways to produce MGMCs: precipitation of a dendritic crystalline phase from the glass matrix (in-situ composites) [3-6]; casting of a glass-forming alloy and reinforcements such as crystalline particles and fibers (exsitu composites) $[7,8]$; and production of a nanocrystalline phase in metallic glass $[9,10]$. Among these composites, the most common are the in-situ composites developed by copper-mold-suction casting due to the convenient and easy fabrication.

The in-situ MGMCs usually exhibit macroscopic plasticity upon quasi-static loading at room temperature due to the interaction between the dendrites and the multiple shear bands [3-6]. However, when they are subjected to high strain-rate loading, the situation may be different. The information on the mechanical properties of the materials upon dynamic loading is of great importance, which can be effectively applied to strategic fields, such as defense, aerospace, and precision machinery $[11,12]$. In spite of the several investigations conducted on the dynamic compression behavior of in-situ MGMCs [11-16], agreements have been rarely reached on the deformation mechanisms. Qiao et al. [11] have found that in-situ Zr-based MGMCs exhibited ultrahigh strength and considerable plasticity upon quasi-static compressive loading, but failed abruptly with brittleness upon dynamic compression due to the absence of multiple shear bands. Similar results of a Zr-based MGMC were also reported by Chen et al. [13]. However, Jeon et al. [12] have reported that the maximum fracture strain of Zr-based MGMCs upon dynamic loading amounts to as high as $10 \%$. Recently, Wang et al. [14] have found that the total strain of an in-situ Ti-based MGMC upon dynamic loading is over $7 \%$. Similarly, a Ti-based MGMC, exhibiting distinguished work-hardening capability upon dynamic compressive loading, was investigated, and the fracture strain was up to $10.6 \%$ [15]. Additionally, considerable plasticity was available, when in-situ Tibased dendrite/metallic glass matrix composites were subjected to the dynamic compression [16]. The inconsonant results motivate more investigations on the dynamic compression behavior of in-situ MGMCs, which can help to get a better understanding of deformation and fracture mechanisms.short milling time.

Ti alloys are widely used in aerospace field owing to their unique combination of physical and mechanical properties, such as low density and high specific stiffness and strength [17]. Based on this, a new kind of in-situ Ti-based metallic glass matrix composite is fabricated by copper-mold-suction casting in this study. Quasi-static and dynamic compression experiments are conducted to explore the deformation and fracture mechanisms of the present in-situ MGMC. Dependence of the mechanical properties on microstructures and loading rates is investigated.

\section{Experimental}

An ingot of a nominal composition: $\mathrm{Ti}_{46} \mathrm{Zr}_{20} \mathrm{~V}_{12} \mathrm{Cu}_{5} \mathrm{Be}_{17}$ (at. \%) was prepared by arc-melting a mixture of $\mathrm{Ti}, \mathrm{Zr}, \mathrm{V}, \mathrm{Cu}$, and Be with purity higher than $99.9 \%$ under a Ti-gettered argon atmosphere. In order to ensure homogeneity, the master alloy ingot was re-melted at least four times. Then, the rod-like samples with $3 \mathrm{~mm}$ in diameter and $85 \mathrm{~mm}$ in length were fabricated by suctioning the molten ingot into a copper mold. The cross sections of the rods were polished, and etched by a solution of $40 \mathrm{~mL} \mathrm{HF}, 20 \mathrm{~mL} \mathrm{HNO}_{3}, 40 \mathrm{~mL} \mathrm{HCl}$, and $200 \mathrm{~mL} \mathrm{H}_{2} \mathrm{O}$. "Corresponding Author: Dr. Junwei Qiao, Laboratory of Applied Physics and Mechanics of Advanced Materials, College of Materials Science and Engineering, Taiyuan University of Technology, Taiyuan 030024, China ; E-mail: qiaojunwei@ gmail.com

Citation: Chu MY, Jiao ZM, Zhang ZH, Wang ZH, Yang HJ, et al. (2015) Quasistatic and Dynamic Compression Behaviors of an in-situ Ti-based Metallic Glass Matrix Composite. Int J Metall Mater Eng 1: 106. doi: http://dx.doi. org/10.15344/2455-2372/2015/106

Copyright: ( 2015 Chu et al. This is an open-access article distributed under the terms of the Creative Commons Attribution License, which permits unrestricted use, distribution, and reproduction in any medium, provided the original author and source are credited. 
Citation: Chu MY, Jiao ZM, Zhang ZH, Wang ZH, Yang HJ, et al. (2015) Quasi-static and Dynamic Compression Behaviors of an in-situ Ti-based Metallic Glass Matrix Composite. Int J Metall Mater Eng 1: 106. doi: http://dx.doi.org/10.15344/2455-2372/2015/106

Page 2 of 5

The scanning-electron microscopy (SEM) was employed to investigate the microstructure. The phases of the samples were analyzed by the $\mathrm{X}$-ray diffraction (XRD). Uniaxial quasi-static compressions were performed on cylindrical samples with $3 \mathrm{~mm}$ in length under a strain rate of $5 \times 10^{-4} \mathrm{~s}^{-1}$ at room temperature. The dynamic compressive loadings were conducted at ambient temperature on samples with an aspect ratio of 1:1 using a split Hopkinson pressure bar (SHPB) apparatus. An SHPB apparatus was illustrated in Figure 1[11], which consisted of input and output bars, which were made of high strength steels. The sample was located between the input and output bars. The striker bar was launched from a gas gun towards the input bar. A compressive stress pulse was generated, when the input bar was impacted by the striker bar, which would travel along the input bar towards the sample and subject it to the required stress levels. A portion of the pulse was reflected back into the input bar, while the remaining pulse was transmitted into the output bar. The input and output bars were both mounted with strain gages at midway points along the length of the bars to capture the strain signals associated with the waves when they passed by. Therefore, both the compressive strain rate, $\dot{\varepsilon}$, and the strain, $\varepsilon$, expressed as a function of time, $t$, could be obtained from the reflected wave, $\varepsilon_{r}(t)$, and the stress, $\sigma$, also expressed as a function of time, $t$, could be obtained from the transmitted wave, $\varepsilon t(t)$. The formulas were expressed as follows:

\section{Results and Discussion}

Figure 2 shows the typical SEM image of the microstructure of the in-situ composite. It can be seen that dendrites are homogeneously distributed within the featureless and continuous glass matrix. The volume fraction of the dendrites is approximately $60 \%$, and the average diameter of dendritic arms is about $0.5 \mu \mathrm{m}$. During cooling of the melt from high temperature, the embedding dendrites are formed by nucleation and dendritic growth of the bcc $\beta$-Ti phase, followed by the solidification of the remaining liquid alloy. The XRD pattern of the composite, shown in the inset, indicates that only the $\beta$-Ti crystalline phase with a body-centered-cubic (bcc) structure can be detected. The sharp diffraction peaks of the $\beta$-Ti phase are superimposed on the broad diffuse scattering amorphous maxima, which is in agreement with the SEM result, further identifying the dual-phase structure.

Figure 3(a) displays the quasi-static compressive engineering stress-strain curve of the composite. It can be seen that the yielding strength is about 1,600 MPa. After yielding, a work-hardening behavior prevails until the final fracture takes place, accompanied by the ultimate strength of $1,980 \mathrm{MPa}$ and the fracture strain of about $8.8 \%$. Analogical phenomena are found previously in in-situ Ti-based MGMCs [14-16]. There are several empirical formulas to explain the

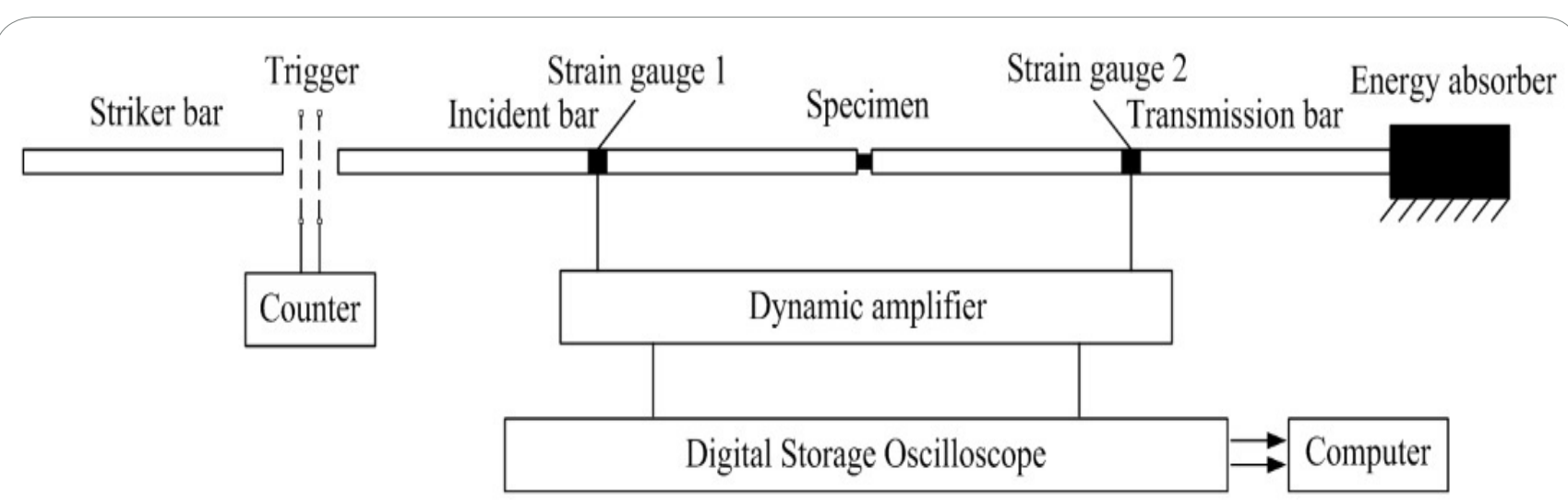

Figure 1: Schematic diagram of the Split Hopkinson Pressure Bar (SHPB) [11].

$$
\begin{aligned}
& \dot{\varepsilon}=-\frac{2 C_{b}}{L_{s}} \varepsilon_{t}(t) \\
& \varepsilon=-\frac{2 C_{b}}{L_{s}} \int_{0}^{t} \varepsilon_{r}(t) d t \\
& \sigma=\frac{A_{b} E_{b}}{A_{s}} \varepsilon_{t}(t)
\end{aligned}
$$

Where $A, L, E$, and $C$ were the cross section area, length, elastic modulus, and elastic wave velocity, respectively. The $s$ and $b$ represented the sample and pressure bar, respectively. Therefore, the stress-strain curves upon dynamic compression could be obtained by eliminating the time parameter, t. More descriptions of the dynamic compression process could be found elsewhere $[11,18]$. After the quasi-static and dynamic compressions, lateral surfaces and fracture surfaces of the deformed samples were investigated by SEM to identify the deformation and fracture mechanisms.

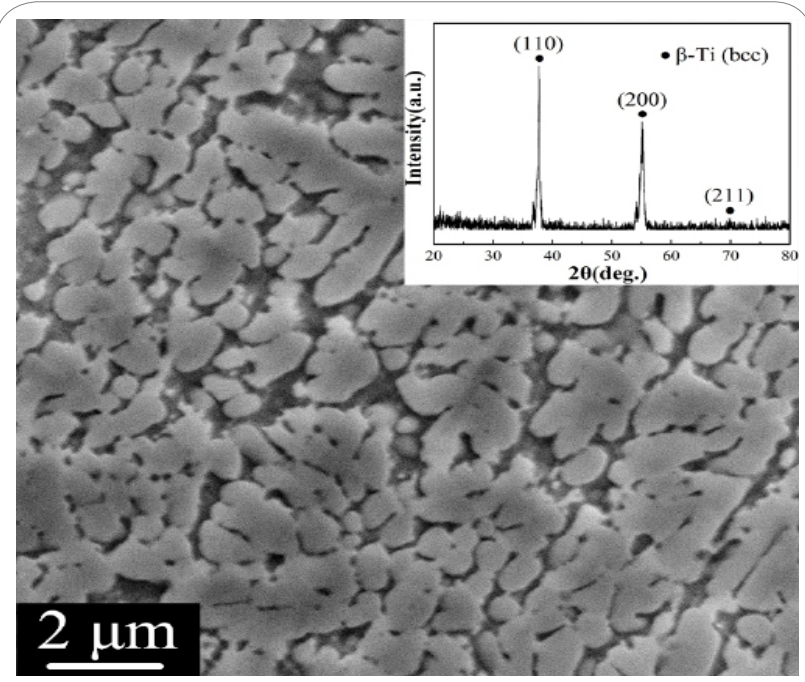

Figure 2: The microstructure of the composite and the corresponding XRD pattern shown in the inset. 
Citation: Chu MY, Jiao ZM, Zhang ZH, Wang ZH, Yang HJ, et al. (2015) Quasi-static and Dynamic Compression Behaviors of an in-situ Ti-based Metallic Glass Matrix Composite. Int J Metall Mater Eng 1: 106. doi: http://dx.doi.org/10.15344/2455-2372/2015/106

work-hardening capacity of metallic materials, among which, the Hollomon equation [19] is widely used, since it goes well with the uniform plastic deformation stage in the true stress-strain curve of metallic materials. The equation is expressed as follows:

$$
S=K \varepsilon^{n}
$$

Where $\mathrm{S}$ is the true stress, $\mathrm{K}$ is the strengthening coefficient, $\varepsilon$ is the true strain, and $n$, indicating the ability of resistance to continuous plastic deformation, is the work-hardening exponent. The workhardening exponent, $\mathrm{n}$, of the present composite, calculated based on the Hollomon equation, is about 0.15 , which is several times larger than that of Ti-6Al-4V alloys (0.03 0.06), extensively applied in the field of aerospace [20]. Generally, the work-hardening exponent, $\mathrm{n}$, is inversely proportion to the yielding strength, $\sigma \mathrm{y}$, which can be identified by the comparison between the present results and the previous studies by Qiao et al. [21] $(\mathrm{n}=0.25, \sigma y=1,300 \mathrm{MPa})$. For the composite upon quasi-static loading, the inconsistent deformation exists for the dendrites and glass matrix, due to the mismatch of Young's modulus and yielding strengths between the two phases $[3,22,23]$. Initially, the applied stress is relatively low, so both of the crystalline dendrites and the glass matrix deform elastically, the composite, therefore, goes through the elastic stage. When the stress increases continually, a critical point will be approached, where the dendrites begin to yield, and work-hardening appears simultaneously.

However, owing to possessing higher strength than the dendrites, the glass matrix is still at the stage of the elastic deformation. Meanwhile, primary shear bands would initially nucleate near the interface of the two phases, which propagate along the favorable direction. When the propagation is hindered by the crystalline dendrites, the shear bands either are arrested by the crystalline phases or bypass the barriers wriggly $[1,3,21]$. Consequently, the multiplication of shear bands prevails within the glass matrix, and plasticity of the composite is greatly improved. Therefore, the work hardening of the crystalline dendrites is responsible for the macroscopic plasticity of the composite, high strength of the glass matrix accounts for the large yielding and ultimate strengths of the composite. When the crystalline dendrites can not undertake more plastic deformation, further deformation of the composite would be dominated by the glass matrix. Unfortunately, the monolithic amorphous alloys exhibit strain-softening rather than work-hardening after yielding [24]. As a result, the final fracture takes place along the maximum shear stress direction.

Figure 3(b) exhibits the engineering stress-strain curves of the composites upon dynamic compressive loading with different strain rates. Unlike the quasi-static case, the samples begin to lose their load-carrying capacity, once the maximum stress is approached, i.e., all of them have a fracture without obvious macroscopic plasticity. The ultimate strength of the samples ranges from 1,710 MPa to 2,030 MPa. Zener and Hollomon [25] proposed a constitutive equation for the coupled strain rate / temperature dependence of irons and steels as follows:

$$
\sigma / \sigma_{0} \mu\left\{\left[(d \varepsilon / d t) /(d \varepsilon / d t)_{0}\right] \exp (Q / R T)\right\}^{r}
$$

Where $\sigma_{0}$ and $(d \varepsilon / d t)_{0}$ are reference flow stress and strain rate, respectively, $Q$ represents the activation energy, $R$ indicates the gas constant, $T$ refers to the temperature, and $r$ is an experimental constant. It can be seen that $\sigma$ increases with the larger strain rate, $d \varepsilon / d t$, at a given temperature. Additionally, the crystalline alloys, for example, Ti-6Al-4V alloys , usually exhibit increased yielding and
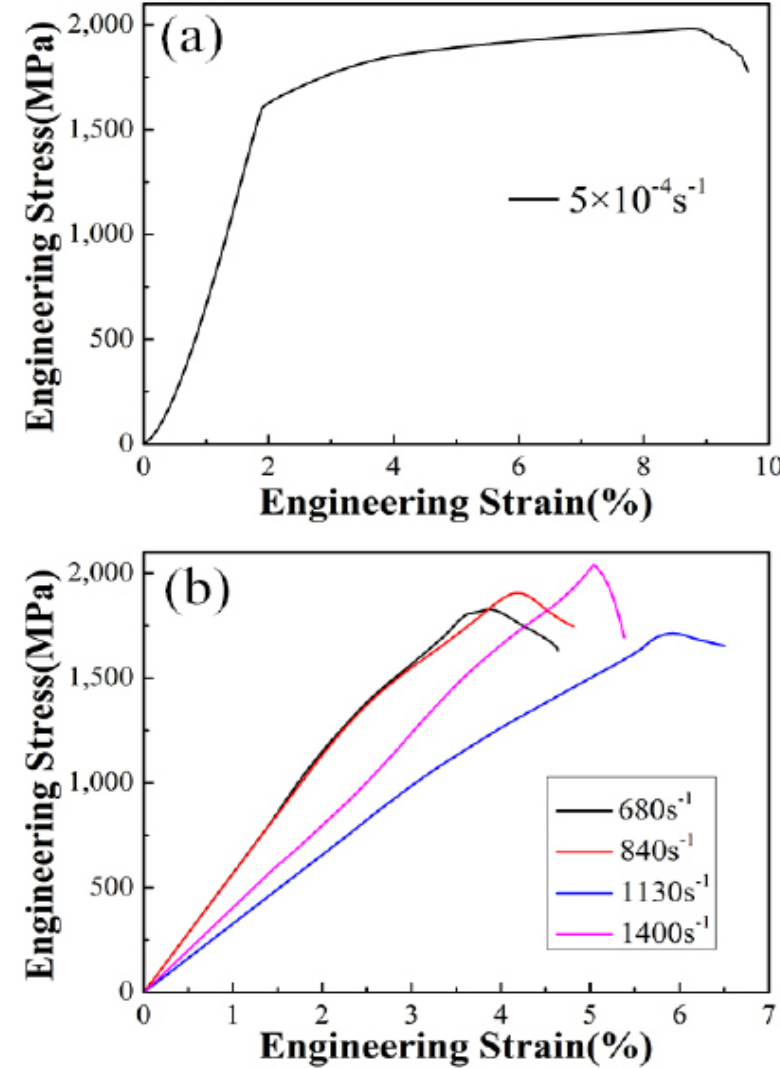

Figure 3: The engineering stress-strain curves of the composites upon (a) quasi-static compression and (b) dynamic compression.

ultimate strengths upon dynamic loading compared to quasi-static loading [18]. However, a weak dependence of the strength on the strain rate is generally obtained for monolithic BMGs and in-situ MGMCs $[11,26]$. The phenomena can be explained by that the above analysis of the dependence of the deformation of the crystalline alloys on the strain rate is based on the dislocation mechanism, whereas the dislocations are absent in BMGs. And due to the presence of the two phases, the situation becomes more complicated for the in-situ MGMCs upon dynamic compression.

For the present composites upon dynamic loading, there is not sufficient time for the generation of multiple shear bands. Once initiated, the shear bands would propagate rapidly, leading to the decrease of the resistance to fracture. Therefore, all the composites fail with brittleness. Spaepen [27] has concluded the softening mechanism during inhomogeneous deformation for BMGs: if there is a lowering of viscosity in the shear bands, there must be an increase of the free volumes.

$$
\dot{\gamma}=\chi \exp \left(-\frac{\alpha V^{*}}{V_{f}}\right) \exp \left(-\frac{\Delta G^{m}}{R T}\right) \sinh \left(\frac{\tau \lambda \Omega}{2 R T}\right)
$$

where - is the shear strain rate, $\chi$ is a factor associated with the amount of the flow units, $\alpha$ is a constant between 1 and $1 / 2, V^{*}$ is the effective hard-sphere size of atoms, $V_{f}$ is the average free volume of an atom, $\Delta \mathrm{G}^{\mathrm{m}}$ is the thermal activation energy, $R$ is the gas constant, $T$ is the absolute temperature, and $\Omega$ is the molar atomic volume. The value of $\lambda$ is in the range of $0 \sim 1$. From Eq. (6), it can be deduced that the higher shear strain rate would create more average free volumes, which facilitate the propagation of shear bands. For the insitu MGMCs upon dynamic loading, the crystalline dendrites can not effectively impede the prompt propagation of shear bands. As a 
Citation: Chu MY, Jiao ZM, Zhang ZH, Wang ZH, Yang HJ, et al. (2015) Quasi-static and Dynamic Compression Behaviors of an in-situ Ti-based Metallic Glass Matrix Composite. Int J Metall Mater Eng 1: 106. doi: http://dx.doi.org/10.15344/2455-2372/2015/106

consequence, individual mature shear bands are available upon dynamic loading. The shear cracks would be generated and propagate along these mature shear bands, inducing the abrupt fracture of the composites. Therefore, all the composites fail without obvious macroscopic plasticity.

In order to better understand the deformation mechanisms of the current composite, it is necessary to investigate the fractographs of the composites. Figure 4(a) shows the lateral surface of the sample fractured upon quasi-static compression. The fracture plane inclines about $45^{\circ}$ with respect to the compressive loading direction. Usually, the shearing angle of metallic glasses is less than $45^{\circ}$ [28-30]. However in terms of in-situ MGMCs, when subjected to applied stress, the fracture would proceed along the maximum shear stress direction (about $45^{\circ}$ with the loading direction) due to the presence of crystalline dendrites. Figure 4(b) displays the magnified image for the lateral surface of the deformed sample. Profuse shear bands are distributed on the lateral surface near the fracture plane, indicating macroscopic plasticity of the sample. It can be seen that the shear bands are mainly in two different directions, which are basically perpendicular to each other. Several microcracks, indicated by the red arrows, can be also observed, which are formed along the shear bands due to severe plastic deformation. Figure 4(c) shows dense shear bands parallel to each other with an average spacing of $0.8 \mu \mathrm{m}$. Figure $4(\mathrm{~d})$ presents the typical fracture surface of the composite. Few vein patterns can be found, and abundant resolidified liquid droplets, associated with the adiabatic heating [28], prevail on the whole facture surface. Figure 5(a) illustrates the lateral surface of the deformed sample, subjected to the dynamic compression. A piece of the fragment has broken away from the whole specimen, as indicated by the red ellipse, partly demonstrating that the specimen fracture with brittleness. Figure $5(\mathrm{~b})$ presents the magnified image for the lateral surface of the deformed
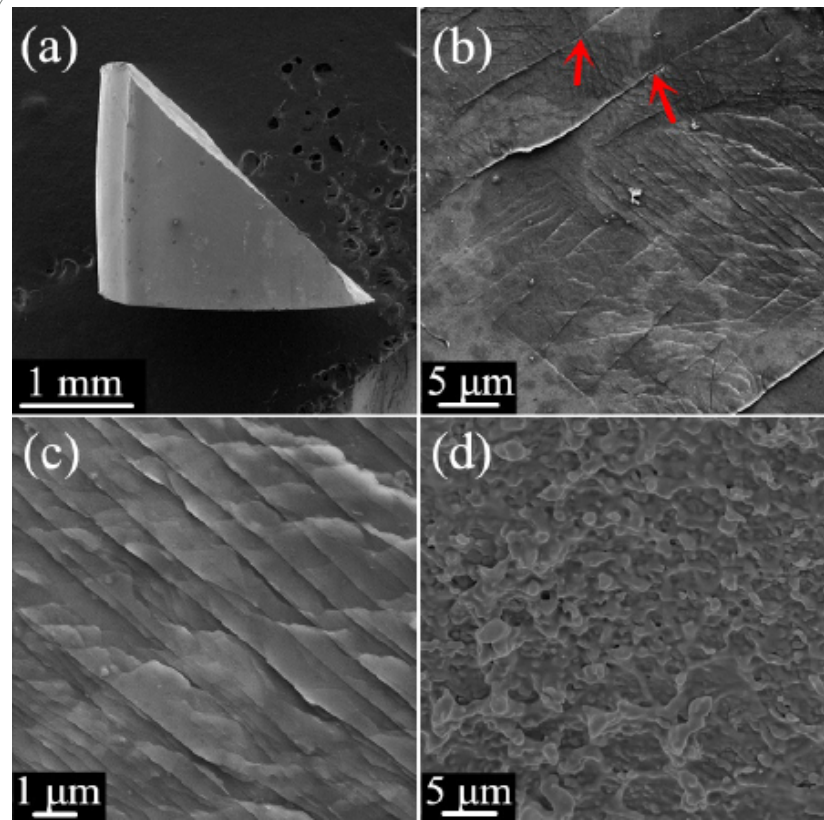

Figure 4: The fractographs of the composite upon quasi-static compression. (a) The lateral surface of the whole deformed composite; (b) the magnified image for the lateral surface of the deformed sample, multiple shear bands and several microcracks, indicated by the red arrows, can be seen; (c) dense shear bands with a spacing of $0.8 \mu \mathrm{m}$; (d) the fracture surface of the composite.
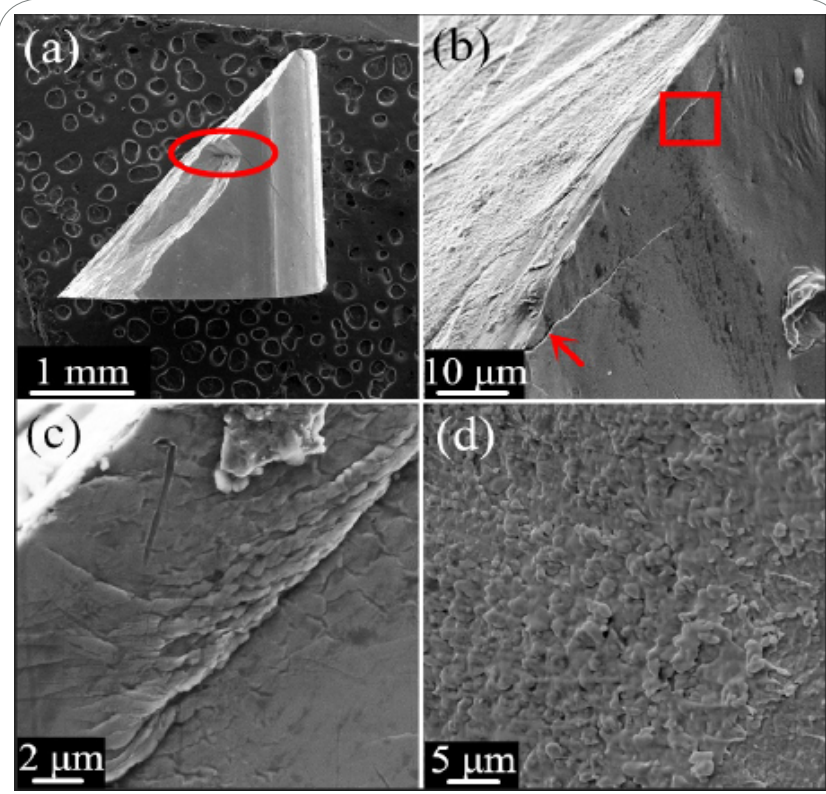

Figure 5: The fractographs of the composite upon dynamic compression. (a) The lateral surface of the whole deformed composite, from which, a piece of fragment, indicated by the red ellipse, has come away; (b) the magnified image for the lateral surface of the deformed sample, only primary shear bands and microcrack, indicated by the red arrow, can be seen; (c) the higher magnification of the area marked by the red rectangular in Figure 5(b), showing the details of the shear band; (d) the fracture surface of the composite.

sample. There are no traces of multiple shear bands, and only primary shear bands along the fracture plane can be found. As the quasi-static case, microcrack, indicated by the red arrow, comes into being along the primary shear band. The area, marked by the red rectangular in Figure 5(b), is magnified and displayed in Figure 5(c), showing the details of the shear bands. Figure 5(d) shows the fracture surface of the sample. Compared with that upon quasi-static compression, the dynamic fracture surface is much smoother. According to the investigation by Chen et al. [13], the increased strain rate leading to the decrease in the shear band toughness, $K_{c}$, which measures the critical energy dissipated in the shear bands. Upon quasi-static loading, the larger shear band toughness, $K$, denotes higher energy dissipation in the shear bands, resulting in the higher temperature rise for the specimen in the instant of fracture. Therefore, more resolidified droplets can be observed on the fracture surface of the sample upon quasi-static compression.

\section{Conclusion}

An in-situ Ti-based metallic glass matrix composite with the composition of $\mathrm{Ti}_{46} \mathrm{Zr}_{20} \mathrm{~V}_{12} \mathrm{Cu}_{5} \mathrm{Be}_{17}$ (at. \%), containing homogeneously distributed $\beta$-Ti dendrites within the glass matrix, was fabricated by copper-mold-suction casting. Upon quasi-static compression, macroscopic plasticity was available for the composite, characterized by multiple shear bands on the lateral surface and abundant droplets on the fracture surface in the micro. However, when subjected to the dynamic loading, all the samples fractured with brittleness, due to the insufficient time for multiple shear bands to be generated. The fractographs of the composites upon quasi-static and dynamic compressions were observed and compared.

\section{Competing Interests}

The author declare that he has no competing interests exits. 
Citation: Chu MY, Jiao ZM, Zhang ZH, Wang ZH, Yang HJ, et al. (2015) Quasi-static and Dynamic Compression Behaviors of an in-situ Ti-based Metallic Glass Matrix Composite. Int J Metall Mater Eng 1: 106. doi: http://dx.doi.org/10.15344/2455-2372/2015/106

Page 5 of 5

\section{Author Contributions}

All the authors substantially contributed to the study conception and design as well as the acquisition and interpretation of the data and drafting the manuscript.

\section{Acknowledgements}

J.W.Q. would like to acknowledge the financial support of National Natural Science Foundation of China (No. 51371122), the Program for the Innovative Talents of Higher Learning Institutions of Shanxi (2013), and the project of State Key Laboratory of Explosion Science and Technology (Beijing Institute of Technology), and the project number is KFJJ16-07. M. H.J.Y. would like to acknowledge the financial support from the National Natural Science Foundation of China (No. 51401141), State Key Lab of Advanced Metals and Materials (No. 2013-Z03), and the Youth Science Foundation of Shanxi Province, China (No. 2014021017-3). Z.H.W. would like to acknowledge the National Natural Science Foundation of China (No. 11390362).

\section{References}

1. Miller MK, Liaw PK (2007) Bulk Metallic Glasses, Springer, New York.

2. Schuh CA, Hufnagel TC, Ramamurty U (2007) Mechanical Behavior of Amorphous Alloys. Acta Materialia 55: 4067-4109.

3. Hays CC, Kim CP, Johnson WL (2000) Microstructure Controlled Shear Band Pattern Formation and Enhanced Plasticity of Bulk Metallic Glasses Containing in situ Formed Ductile Phase Dendrite Dispersions. Physical Review Letters 84: 2901-2904.

4. Hofmann DC, Suh JY, Wiest A, Duan G, Lind ML, et al. (2008) Designing Metallic Glass Matrix Composites with High Toughness and Tensile Ductility. Nature 451: 1085-1089.

5. Qiao JW, Sun AC, Huang EW, Zhang Y, Liaw PK, Chuang CP (2011) Tensile Deformation Micromechanisms for Bulk Metallic Glass Matrix Composites: From Work-hardening to Softening. Acta Materialia 59: 4126-4137.

6. Qiao JW (2013) In-situ Dendrite / Metallic Glass Matrix Composites: A Review. Journal of Materials Science and Technology 29: 685-701.

7. Dandliker RB, Conner RD, Johnson WL (1998) Melt Infiltration Casting of Bulk Metallic-glass Matrix Composites. Journal of Materials Research 13: 2896-2901.

8. Xue YF, Cai HN, Wang L, Wang FC, Zhang HF (2007) Strength-improved Zrbased Metallic Glass / Porous Tungsten Phase Composite by Hydrostatic Extrusion. Applied Physics Letters 90: 081901.

9. Calin M, Eckert J, Schultz L (2003) Improved Mechanical Behavior of Cu-Tibased Bulk Metallic Glass by in situ Formation of Nanoscale Precipitates. Scripta Materialia 48: 653-658.

10. Kim HS (2003) Strengthening Mechanisms of Zr-based Devitrified Amorphous Alloy Nanocomposites. Scripta Materialia 48: 43-49.

11. Qiao JW, Feng P, Zhang Y, Zhang QM, Liaw PK, et al. (2010) Quasi-static and Dynamic Deformation Behaviors of in-situ Zr-based Bulk-metallicglass-matrix Composites. Journal of Materials Research 25: 2264-2270.

12. Jeon C, Kang M, Kim CP, Kim HS, Lee S (2013) Quasi-static and Dynamic Compressive Deformation Behaviors in Zr-based Amorphous Alloys Containing Ductile Dendrites. Materials Science and Engineering: A 579: 77-85.

13. Chen JH, Jiang MQ, Chen Y, Dai LH (2013) Strain Rate Dependent Shear Banding Behavior of a Zr-based Bulk Metallic Glass Composite. Materials Science and Engineering: A 576: 134-139.

14. Wang YS, Hao GJ, Qiao JW, Zhang Y, Lin JP (2014) High Strain Rate Compressive Behavior of Ti-based Metallic Glass Matrix Composites. Intermetallics 52: 138-143.

15. Qiao JW, Ye HY, Wang YS, Pauly S, Yang HJ, et al. (2013) Distinguished Work-hardening Capacity of a Ti-based Metallic Glass Matrix Composite upon Dynamic Loading. Materials Science and Engineering: A 585: 277 280 .
16. Qiao JW, Chu MY, Cheng L, Ye HY, Yang HJ, et al. (2014) Plastic Flows of in-situ Metallic Glass Matrix Composites upon Dynamic Loading. Materials Letters 119: 92-95. Banerjee D, Williams JC (2013) Perspectives on Titanium Science and Technology. Acta Materialia 61: 844-879.

17. Banerjee D, Williams JC (2013) Perspectives on Titanium Science and Technology. Acta Materialia 61: 844-879.

18. Armstrong RW, Walley SM (2008) High Strain Rate Properties of Metals and Alloys. International Materials Reviews 53: 105-128.

19. Hollomon JH (1945) Tensile Deformation. Transaction of American Institute of Mining, Metallurgical, and Petroleum Engineers 162: 268-290.

20. Sen I, Tamirisakandala S, Miracle DB, Ramamurty U (2007) Microstructural Effects on the Mechanical Behavior of B-modified Ti-6Al-4V alloys. Acta Materialia 55: 4983-4993.

21. Qiao JW, Zhang Y, Liaw PK, Chen GL (2009) Micromechanisms of Plastic Deformation of a Dendrite/Zr-based Bulk-metallic-glass Composite. Scripta Materialia 61: 1087-1090.

22. Fan C, Ott RT, Hufnagel TC (2002) Metallic Glass Matrix Composite with Precipitated Ductile Reinforcement. Applied Physics Letters 81: 1020-1022.

23. Orellana T, Tejado EM, Funke C, Fütterer W, Riepe S, et al. (2015) How do Impurity Inclusions Influence the Mechanical Properties of Multicrystalline Silicon? Int J Metall Mater Eng 1: 101.

24. Bei H, Xie S, George EP (2006) Softening Caused by Profuse Shear Banding in a Bulk Metallic Glass. Physical Review Letters 96: 105503.

25. Zener C, Hollomon JH (1944) Effect of Strain Rate upon Plastic Flow of Steel. Journal of Applied Physics 15: 22-32.

26. Bruck HA, Rosakis AJ, Johnson WL (1996) The Dynamic Compressive Behavior of Beryllium Bearing Bulk Metallic Glasses. Journal of Materials Research 11: 503-511.

27. Spaepen F (1977) A Microscopic Mechanism for Steady State Inhomogeneous Flow in Metallic Glasses. Acta Metallurgica 25: 407-415.

28. Liu CT, Healtherly L, Easton DS, Carmichael CA, Schneibel JH, et al (1998) Test Environments and Mechanical Properties of Zr-Based Bulk Amorphous Alloys. Metallurgical and Materials Transactions A: Physical Metallurgy and Materials Science 29: 1811-1820.

29. Zhang ZF, Eckert J, Schultz L (2003) Difference in Compressive and Tensile Fracture Mechanisms of Zr59Cu20AI10Ni8Ti3 Bulk Metallic Glass. Acta Materialia 51: 1167-1179.

30. Inoue A, Shen BL (2004) A New Fe-based Bulk Glassy Alloy with Outstanding Mechanical Properties. Advanced Materials 16: 2189-2192. 